recently described rhabdomyosarcoma subtype. Histopathology 2018 73: $514-20$.

13. Miao D, Margolis CA, Gao W, et al. Genomic correlates of response to immune checkpoint therapies in clear cell renal cell carcinoma. Science 2018; 359: 801-6.

DOI: https://doi.org/10.1016/j.pathol.2019.07.004

\section{Innocuous clinical presentation of a SMARCA4-deficient thoracic sarcoma arising in a patient with chronic empyema thoracis}

Sir,

First described in $2015,{ }^{1}$ SMARCA4-deficient thoracic sarcoma (SMARCA4-DTS) is a recently recognised distinct tumour entity with less than 60 cases reported to date. ${ }^{1-5}$ They usually occur as large (median size $\sim 10 \mathrm{~cm}$ ), compressive, and locally infiltrating intra-thoracic masses in adult male smokers (median age at diagnosis 48 years, ranging from 27 to 90 years), with their epicentres located in the mediastinum or pleura/chest wall, and rarely within the lung. ${ }^{5}$ More than three-quarters of patients present with metastatic disease. ${ }^{5}$ The histopathology is of a high grade undifferentiated tumour with variably epithelioid or rhabdoid morphology and encompasses a wide range of differential diagnoses depending upon the site of origin. The tumours respond poorly to multimodality therapy, and most patients succumb to disease within 5-7 months of presentation. ${ }^{5}$ In stark contrast to the typically aggressive presentation of SMARCA4-DTS, we highlight an unusual clinical scenario wherein this tumour developed insidiously in the intercostal drain (ICD) insertion site of a patient suffering from chronic empyema thoracis

A 60-year-old man, light smoker ( $\sim 2-3$ beedis/day), and a farmer by occupation, presented with sudden onset, progressively increasing breathlessness. A right ICD was placed at a private medical centre that drained $\sim 250 \mathrm{~mL}$ of purulent material/day. Chest radiograph done at the time of presentation to our hospital (10 days after ICD insertion) showed presence of a multiloculated hydropneumothorax on the right side with ICD in situ (Fig. 1A).

Microbiological cultures of the pus grew more than three colonies of Gram negative bacteria, but were negative for Mycobacterium tuberculosis by GeneXpert assay. Pleural fluid cytology showed mainly polymorphs and few mesothelial cells. His serum calcium levels were within normal limits. Computed tomography (Fig. 1B-D) performed after one month showed right empyema with smooth pleural thickening and enhancement with draining catheter in situ. Lung window sections revealed bilateral apical pleural bullae and interspersed emphysematous changes in both lungs. No cavities, consolidations, mediastinal lymphadenopathy, or parenchymal mass lesions were seen. The discharge gradually reduced with time. Two months after ICD placement, there was accidental removal of the same at home and patient presented with pus discharging sinus at the ICD insertion site. A right thoracotomy was planned for thoracic window creation. Intraoperatively, loculated thick pus was seen in the posterior aspect of right pleural cavity with thickened pleura. No suspicious mass lesion was seen. Nearly $300 \mathrm{~mL}$ of pus was drained and an open thoracic window was created after cutting the 6th and 7 th ribs just anterior to the mid-axillary line. Repeat cultures were
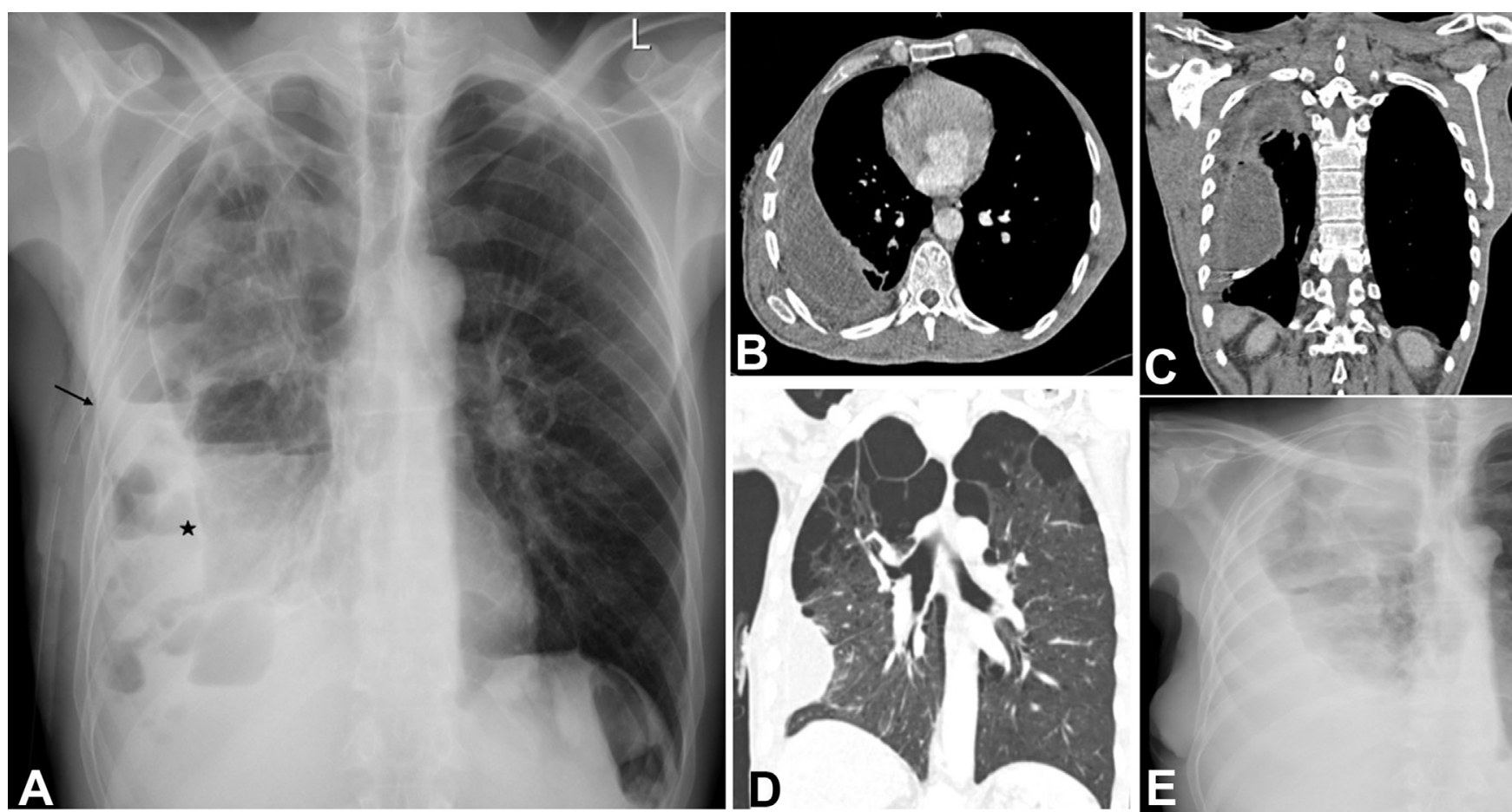

Fig. 1 (A) Chest radiograph 10 days after insertion of intercostal drainage tube (ICD) showed persistent multiloculated right hydropneumothorax with multiple air-fluid levels (*) and ICD in situ (arrow). (B) Axial and (C) coronal mediastinal window computed tomography sections depict right empyema with rib crowding and smooth uniformly enhancing pleural thickening. (D) Coronal lung window sections depict bilateral apical pleural bullae and interspersed emphysematous changes. (E) Follow-up chest radiographs show worsening of right effusion. 
negative for Mycobacterium. The patient was well for the next 5-6 months until he again developed shortness of breath. Examination revealed non-draining thoracic window site and chest radiograph showed increasing right pleural effusion (Fig. 1E). The patient was re-admitted for refreshing the thoracic window. Intraoperatively, a $3 \times 2 \mathrm{~cm}$ 'hypertrophic granulation tissue-like' mass was found at the previous skin flap. This was excised completely and sent for histopathological examination (Fig. 2). The skin flap was refashioned and the ICD tube was placed. The patient was discharged on the 5th post-operative day in a stable condition. However, he suddenly collapsed and died at home 2 days later. An autopsy was refused.

Histopathology of the 'granulation tissue' revealed a monotonous population of large polygonal rhabdoidphenotype cells with large nuclei and prominent eosinophilic nucleoli arranged in sheets reaching up to the overlying dermis. Frequent mitoses and foci of necrosis were seen. Glandular differentiation, squamous pearls or normal lung parenchyma were not seen. MIB1 labelling index was $\sim 7-$ $10 \%$ in the highest labelling areas. The tumour cells were diffusely immunopositive for vimentin, CD34, MIC2, p53, and SALL4, very focally immunopositive for pan-cytokeratin (AE1/AE3); they were negative for EMA, p40, TTF-1, cytokeratin-7, Hepar-1, desmin, myogenin, CD31, S100, Melan-A, HMB-45, GATA-3, calretinin, WT1, leucocyte common antigen, CD3, CD20, CD79a, CD43, MPO, CD30, CD138, CD38, MUM1, and EBV-LMP1. Tumour cells retained INI-1 protein expression, but showed diffuse loss of SMARCA4 (Brg1), rendering a final diagnosis of SMARCA4DTS arising from chest wall (Fig. 2D).

Characterised by inactivating somatic mutations in SMARCA4 gene encoding for Brg1 protein of the Switch/ Sucrose Non-Fermentable (SWI/SNF) chromatin modifying complex, SMARCA4-DTS belongs to the genetic spectrum of SWI/SNF-deficient neoplasms. ${ }^{1}$ The best studied example of SWI/SNF-deficient neoplasms is the SMARCB1/INI-1 deficient paediatric malignant rhabdoid tumour (MRT) that is characterised by germline SMARCB1 mutations, presentation at a very young age, and an aggressive clinical course. Similar germline SMARCA4 mutations define SMARCA4mutated small cell carcinoma of the ovary, hypercalcaemic type (SCCOHT), ${ }^{6}$ and SMARCA4-mutated infantile pulmonary teratoid tumour. ${ }^{7}$ Although SMARCA4-DTS shares a similar transcriptomic profile to MRT and SCCOHT, unlike the latter, SMARCA4-DTS patients lack germline mutations, present with tumour much later in life, and the tumours harbour complex genomic profiles with frequent TP53 mutations. ${ }^{1}$ Interestingly, somatic SMARCA4 mutations similar to those occurring in SMARCA4-DTS have also been reported in rare subsets of adult high grade epithelial malignancies arising from the endometrium, gastrointestinal tract, head and neck, urinary tract and lung. ${ }^{8}$

SMARCA4-DTS must be a diagnostic consideration in any high grade undifferentiated malignancy arising primarily in the thoracic cavity. Loss/reduction in $\operatorname{Brg} 1$ protein is diagnostic, and in conjunction with concurrent loss/ reduction of SMARCA2/Brm, frequent positivity for CD34, SALL4 and SOX2, and lack of diffuse expression of epithelial markers, ${ }^{5}$ most histological mimics, including the possibilities of epithelioid sarcoma, proximal type, malignant mesothelioma, and haematolymphoid malignancy, which were considered in our patient, are easily excluded. ${ }^{5}$ In a younger female, it may be important to rule out metastasis from a primary ovarian SMARCA4-mutated SCCOHT. ${ }^{6}$ While there was no lung parenchymal involvement in our case, SMARCA4-DTS is usually infiltrative and commonly shows lung involvement. ${ }^{5}$ Especially in cases with lung parenchymal involvement, differentiation from the recently described SMARCA4-deficient
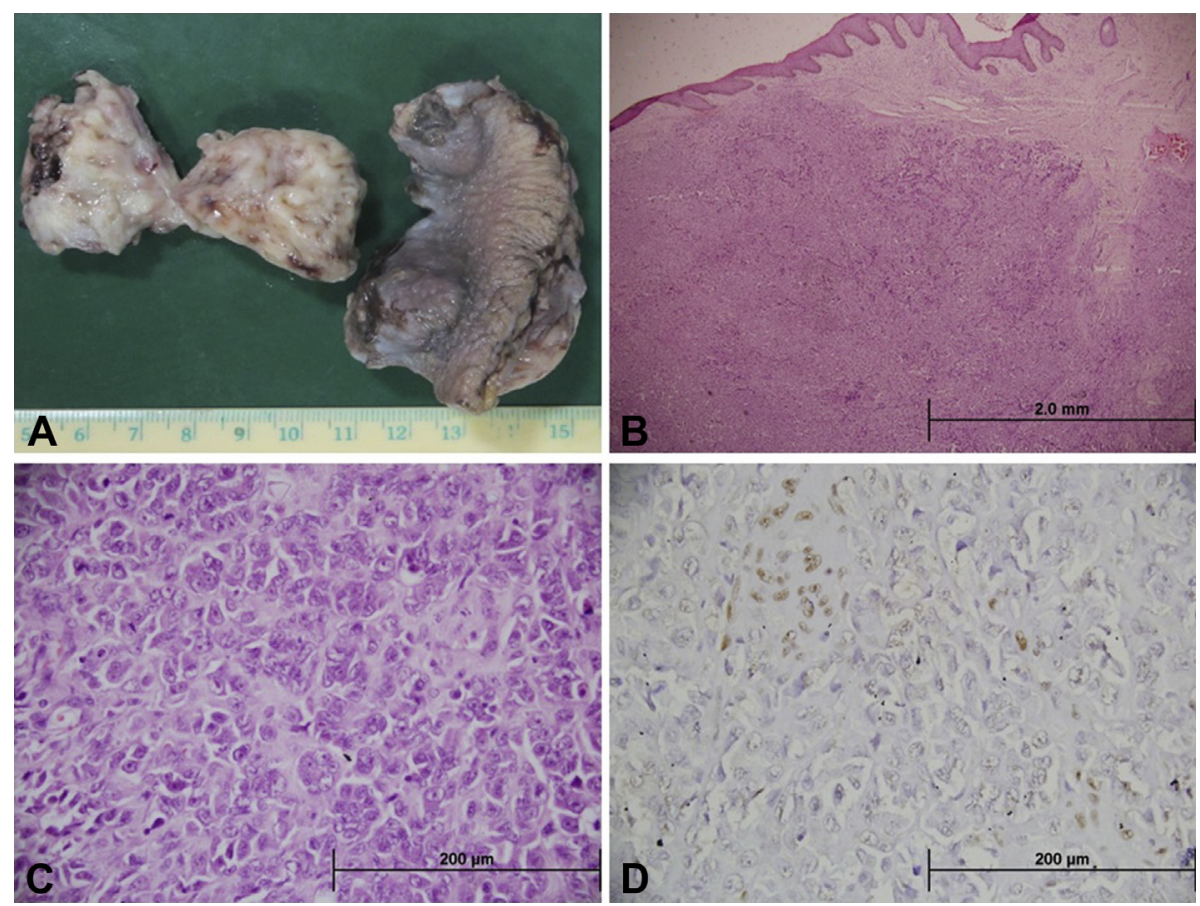

Fig. 2 (A) Gross photograph of the skin covered soft tissue mass removed during surgery for refreshing the thoracic window. (B,C) H\&E stained sections show a high grade undifferentiated tumour composed of monotonous large tumour cells with abundant cytoplasm, round to oval nucleoli and prominent nucleoli arranged in sheets in the dermis. (D) The tumour cells show diffuse loss of SMARCA4/Brg1 expression with endothelial cells serving as internal positive controls. 
non-small cell lung carcinoma (NSCLC) may be challenging. ${ }^{8,9}$ The latter shows striking pleomorphism in contrast to the monotonous appearance of SMARCA4-DTS, is frequently positive for Hepar-1 and cytokeratin-7, and usually shows some degree of glandular differentiation which is always absent in SMARCA4-DTS. ${ }^{8}$ Nevertheless, SMARCA4-DTS and SMARCA4-deficient NSCLC show considerable overlap in their clinicopathological profiles: male preponderance, association with smoking, pattern of metastases, poor clinical outcomes, histomorphology, immunoprofile, and molecular alterations such as concurrent TP53 and KRAS alterations. 5,8 Whether these two tumours represent spectral ends of the same entity needs further clarification.

We document, for the first time, an innocuous presentation of SMARCA4-DTS wherein a small tumour ( $\sim 4 \mathrm{~cm}$ grossly) was incidentally detected in the soft tissue of chest wall around an ICD insertion site in a patient suffering from chronic empyema thoracis of uncertain aetiology for over 10 months. We believe it is unlikely that the SMARCA4-DTS was masquerading as chronic empyema as the initial computed tomography images do not show any mass lesion. The lung parenchyma was completely clear except for smoking related parenchymal changes. Although an autopsy was refused in this patient, the rapidly fatal outcome after development of tumour highlights its aggressiveness. A link with chronic infection/inflammation as an inciting/contributing factor for SMARCA4-DTS has not been noted previously and should be explored.

Conflicts of interest and sources of funding: The authors state that there are no conflicts of interest to disclose.

\section{Aruna Nambirajan ${ }^{1}$, Rajinder Parshad ${ }^{2}$, Ankur Goyal ${ }^{3}$, Mithun N.K. ${ }^{2}$, Deepali Jain}

${ }^{1}$ Department of Pathology, All India Institute of Medical Sciences, New Delhi, India; ${ }^{2}$ Department of Surgery, All India Institute of Medical Sciences, New Delhi, India; ${ }^{3}$ Department of Radiodiagnosis, All India Institute of Medical Sciences, New Delhi, India

Contact Deepali Jain, MD.

E-mail: deepalijain76@gmail.com

1. Le Loarer F, Watson S, Pierron G, et al. SMARCA4 inactivation defines a group of undifferentiated thoracic malignancies transcriptionally related to BAF-deficient sarcomas. Nat Genet 2015; 47: 1200-5.

2. Yoshida A, Kobayashi E, Kubo T, et al. Clinicopathological and molecular characterization of SMARCA4-deficient thoracic sarcomas with comparison to potentially related entities. Mod Pathol 2017; 30: 797-809.

3. Sauter JL, Graham RP, Larsen BT, et al. SMARCA4-deficient thoracic sarcoma: a distinctive clinicopathological entity with undifferentiated rhabdoid morphology and aggressive behavior. Mod Pathol 2017; 30: $1422-32$.

4. Kuwamoto S, Matsushita M, Takeda K, et al. SMARCA4-deficient thoracic sarcoma: report of a case and insights into how to reach the diagnosis using limited samples and resources. Hum Pathol 2017; 70: 92-7.

5. Perret R, Chalabreysse L, Watson S, et al. SMARCA4-deficient thoracic sarcomas: clinicopathologic study of 30 cases with an emphasis on their nosology and differential diagnoses. Am J Surg Pathol 2019; 43: 455-65.

6. Witkowski L, Carrot-Zhang J, Albrecht S, et al. Germline and somatic SMARCA4 mutations characterize small cell carcinoma of the ovary, hypercalcemic type. Nat Genet 2014; 46: 438-43.

7. Kock L, Fahiminiya S, Fiset PO, et al. Infantile pulmonary teratoid tumor. N Engl J Med 2018; 378: 2238-40.
8. Agaimy A, Fuchs F, Moskalev EA, et al. SMARCA4-deficient pulmonary adenocarcinoma: clinicopathological, immunohistochemical, and molecular characteristics of a novel aggressive neoplasm with a consistent TTF1(neg)/CK7(pos)/HepPar-1(pos) immunophenotype. Virchows Arch 2017; 471: 599-609.

9. Herpel E, Rieker RJ, Dienemann H, et al. SMARCA4 and SMARCA2 deficiency in non-small cell lung cancer: immunohistochemical survey of 316 consecutive specimens. Ann Diagn Pathol 2017; 26: 47-51.

DOI: https://doi.org/10.1016/j.pathol.2019.05.011

\section{Concordance of p16, $\mathrm{FH}$, and alpha-SMA expression with the fumarate hydratase gene mutational status in sporadic and hereditary piloleiomyomas}

Sir,

Piloleiomyoma (PLM) is a rare cutaneous leiomyoma (CL) arising from arrector pili muscles. It appears as a firm, skincoloured to light brown papule or nodule, distributed over the trunk and extremities, occasionally on the face and neck. ${ }^{1}$ It occurs more often as an isolated cutaneous leiomyoma (ICL), representing a sporadic, non-familial condition. Less frequently, PLM occurs as multiple cutaneous leiomyomatosis (MCL), both in hereditary (H-MCL) and non-hereditary settings (NH-MCL). H-MCL represents the cutaneous hallmark of hereditary leiomyomatosis and renal cell cancer (HLRCC), an autosomal dominant syndrome that affects heterozygous carriers of germinal mutation (MUT) in the $F H$ gene. HLRCC clinically predisposes to MCL (both diffuse and segmental pattern), multiple uterine leiomyomas, renal cell cancer and phaeochromocytomas. Uterine and cutaneous leiomyosarcoma, ovarian mucinous cystadenoma, bladder, breast, adrenal and testicular cancers have been also described in FH MUT individuals; it remains to be established whether these tumours are effectively associated with HLRCC. ${ }^{1,2}$ The genetic basis of H-MCL is well known; conversely, the pathogenic mechanism of non-hereditary PLM occurring in wild-type FH gene carriers is still unclear. $^{1-6}$ As a rule, wild-type FH subjects manifest a solitary CL (ICL). NH-MCL have been reported only in four wild-type $\mathrm{FH}$ subjects, as a segmental pattern, without unequivocal genetic and immunohistochemical studies (Supplementary Table 1, Appendix A). ${ }^{3-6}$ The diffuse pattern of NH-MCL has never been described.

Recent findings suggest that $\mathrm{FH}$ immunoreactivity is conserved in most sporadic PLMs; conversely, CLs of HLRCC patients show negative or weak positive immunostaining for $\mathrm{FH}^{6}{ }^{6}$ It is well known that benign cutaneous smooth muscle tumours, including PLMs, show immunoreactivity for Alpha-smooth muscle actin (Alpha-SMA). Nevertheless, the expression pattern of alpha-SMA in hereditary and sporadic PLMs is unknown. ${ }^{1,2}$ p16 is one of the most common human tumour suppressor genes. There are some data on the expression of p16 in extra-cutaneous leiomyomas, in melanoma and non-melanoma skin cancers; nonetheless, its expression in PLM is currently completely unexplored. 7

We report the clinical and genetic findings of eight cases of sporadic and familial CL in which FH, p16 and alpha-SMA 